\title{
The Sociology of Quantification: Where Are We Now?
}

\author{
Elizabeth Popp Berman and Daniel Hirschman ${ }^{1}$
}

[Preprint of review essay to be published in Contemporary Sociology]

\section{Books reviewed}

Emily Barman, Caring Capitalism: The Meaning and Measure of Social Value

Wendy Espeland and Michael Sauder, Engines of Anxiety: Academic Rankings, Reputation, and Accountability

Deborah Lupton, The Quantified Self

Sally Engle Merry, The Seductions of Quantification: Measuring Human Rights, Gender Violence, and Sex Trafficking

Dawn Nafus, ed., Quantified: Biosensing Technologies in Everyday Life

Gina Neff and Dawn Nafus, Self-Tracking

Cathy O'Neil, Weapons of Math Destruction

Robert Saldin, When Bad Policy Makes Good Politics: Running the Numbers on Health Reform

A decade ago, Wendy Espeland and Mitchell Stevens published an essay titled "The Sociology of Quantification." In it, they wrote that "sociologists have generally been reluctant to investigate [quantification] as a sociological phenomenon in its own right." While accountants, anthropologists, and historians had begun the reflexive study of numbers, "sociologists have paid

\footnotetext{
${ }^{1}$ Department of Sociology, University at Albany, SUNY; and Department of Sociology, Brown University. We thank Diana Graizbord, Zach Griffen, Philip Rocco, and Lisa Stampnitzky for comments on a previous version of this essay.
} 
relatively little attention to the spread of quantification or the significance of new regimes of measurement" (Espeland and Stevens 2008:402).

That has clearly changed. While Google Scholar shows only nine results for the phrase "sociology of quantification" through 2007, the last decade returns 448. This proliferation of scholarship on numbers goes hand in hand with a proliferation of numbers themselves. New technologies have created a "quantified self," and the explosion of the internet has produced "big data". As a senior sociologist recently quipped to one of us, sociology has become quantitative researchers, and qualitative researchers studying quantification. Thus the moment seems ripe for revisiting the sociology of quantification, looking at emerging themes, and seeking signs that a new subfield might be starting to consolidate.

Alas, the news is mixed. Lots of good work is being done. The intellectual space is full of ferment. Yet—and perhaps the fact that we were reviewing books by authors from at least four different disciplines should have clued us in earlier — so far, we seem to be looking at a genre, not a subfield.

Indeed, the very concept of quantification splinters into fragments as one approaches. Are algorithms quantification? Big data? Biosensors? And what's the difference between quantification, classification, and commensuration? While there are commonalities across all of these topics, and across the eight books we read, the sociology of quantification is still very far from having general claims or a common theoretical language. The closest it comes, probably, is the universal reference to Ted Porter's groundbreaking Trust in Numbers (1995).

Yet all is not lost. While the sociology of quantification may lack a well-defined object of study, shared theoretical concepts, and an agreed-upon methodological toolkit, studies that touch on quantification nevertheless cluster around four broad questions, which we use to orient our 
review. The space is crying out for consolidation and synthesis, and while we only gesture in that direction, we hope that in doing so we point to some productive ways of thinking about numbers.

First, what shapes the production of numbers? Here, we are interested in the technopolitical decisionmaking that guides methodological choices - who gets to decide what we quantify and how we do it (on technopolitics, see Hecht 1998). The project of making numbers is itself sociological, with some actors more influential than others and some numbers easier than others to produce. A significant part of the sociology of quantification is simply showing how social, technical, and political factors interact to make stable numbers.

Second, when and how do numbers matter? When does quantification make a difference? Ian Hacking (1982) characterized the early $19^{\text {th }}$ century as experiencing an "avalanche of printed numbers." This avalanche appears to still be rolling along. Activists, investors, and individuals see in numbers the power to reform states, firms, and their very selves through the governance capacity of numbers. And yet, skeptics note that many of the numbers that get produced are promptly ignored, while other numbers may simply justify decisions that would have been made anyway. Much like the broader debate in political science and sociology around the causal power of ideas, it can be difficult to tease out which kinds of numbers matter and when.

Third, how do we govern quantification? How should we govern quantification? Contemporary controversies about quantification range from concerns about the fairness of predictive algorithms to the objectivity of budget estimates, to privacy concerns surrounding the circulation of our most intimate choices, captured and transformed into big data. These controversies raise new and old questions about governance. As with other technical arenas, quantification often raises significant barriers to democratic deliberation. But some of the numbers we discuss are shocking in their simplicity - the calculations underlying the U.S. News 
\& World Report rankings require no complex algorithms, and yet still have the capacity to reshape the field of higher education.

Fourth and finally, how should scholars study quantification? We identified at least three varieties of quantification studies. First, some authors focus on the effects of a particular (often new) genre of quantification. Here, we examine three texts that look at the "quantified self" movement as examples of this approach. Second, some studies compare across different practices of quantification that share some common features or that are mobilized in the same empirical domain. These studies are especially valuable for providing comparative leverage on the questions of how numbers are produced and when they matter. The third approach consists of case studies that situate a single calculative practice inside a deep study of a single field or decisionmaking context. This research substitutes over-time comparison and detailed process tracing for comparative leverage in order to grapple with the power of numbers.

Together, these books point to a vibrant conversation about quantification happening across many different fields. But such conversations have not yet coalesced into a coherent debate. Rather, these works are loosely connected: a genre with recurring motifs, not an integrated literature with coherent terminology and a clear research program.

\section{What Shapes the Production of Numbers?}

Making numbers requires a lot of work. Sociologists are intimately familiar with the day-to-day challenges confronting would-be quantifiers, from crafting question wordings to defining sampling frames, handling missing variables, constructing indices, and all of the other labor involved in making quantitative knowledge claims. Researchers interested in the power of quantification foreground that potentially invisible labor, and investigate how and why we came 
to have the numbers we have. In particular, researchers focus on the experts, politics, and technologies that shape the production of numbers.

Sally Engle Merry's The Seductions of Quantification and Emily Barman's Caring Capitalism tackle this question most directly, though most books in this genre touch on these issues because it is difficult to trace the effects of quantification without understanding its sources. The politics of production contain within-case counterfactuals that showcase how a number that may seem solid could have been different, and thus can help to identify the consequences of particular decisions.

Merry and Barman approach the question with slightly different theoretical orientations and languages, but with similar methods and very compatible findings. Both books compare multiple cases in a single domain: global human rights indicators for Merry; measures of social value for Barman. As an anthropologist, Merry approaches the creation of human rights indicators through comparative ethnographic work sited in the United Nations and U.S. State Department. After a helpful theoretical introduction, Merry analyzes the creation and circulation of indicators that attempt to measure violence against women, human trafficking, and compliance with human rights treaties.

Through these detailed histories, she argues that indicators are produced by communities of shared expertise, embody theories of social change implying particular modes of action, and are shaped by power inequalities among competing experts. She also emphasizes that history matters through "expertise inertia" and "data inertia" - that is, past decisions about who counts as an expert, how experts are trained, what kinds of data are relevant, and what data have actually been collected shape the potential for developing new indicators or reforming existing ones. In Merry's context, as in many comparable cases, experts were relatively limited in their 
capacity to collect new data, and thus existing data sources became major resources, and serious constraints, on the creation of global human rights indicators.

Barman examines six cases of efforts to measure social value of activities for purposes of investment, and finds that "value entrepreneurs" draw on their own forms of expertise (which suggest what to measure), in conjunction with their "communicative goals" (do they want the valuation device to establish legitimacy? show conformity? change behavior? justify a field?), to produce a particular valuation device. The project is organized around understanding how forprofit or nonprofit status and market- or mission-orientation affect the valuation process.

For our purposes, though, what is most interesting about the book is its analysis of how value entrepreneurs draw on their own form of expertise and their communicative goals to build valuation devices. The six case chapters, which cover valuation projects from outcome measurement to socially responsible investing to corporate responsibility, draw on interviews and documents to show how these come together to produce forms of valuation that embody specific ways of seeing the world and are tailored to specific audiences.

Both Merry and Barman emphasize the role of expertise in suggesting what is worth quantifying and in the political process through which a number stabilizes (or fails to do so). Put differently, the politics of quantification are not open and democratic, but closed and technocratic. Experts thus figure prominently in the central challenges of commensuration.

Both authors also show that producing a number means little, unless you convince others to use it. Thinking across the books helps to illustrate the usefulness of their theoretical insights. Expertise and data inertia (Merry's concepts) seem clearly relevant for understanding the production of numbers in Barman's cases. For example, "Inclusive Business" seeks to address poverty in developing countries by including the poor as employees, customers, suppliers or 
distributors, and by stimulating economic growth. It measures impact by drawing on the numbers already produced by development organizations, which focus on various forms of social change. It does not try to measure the financial value of its favored practices for the businesses who participate, although advocates see the potential of being able to do so, because data inertia means that those numbers are not easily accessible.

Similarly, we could ask of Merry's cases: are there "rights entrepreneurs" to parallel Barman's "value entrepreneurs"? Merry shows, for example, that although a United Nations office put a great deal of work into producing human rights indicators, the "project was a technology in search of an audience, a tool kit without a clientele" (181). Without entrepreneurs to make the link between producing the numbers and without clear communicative goals for the use of those numbers, they were left floating in midair—formed, but never effective.

We are also struck by how both Barman and Merry's work show how the political and epistemological choices that are so explicit during struggles to establish a number disappear once it stabilizes and travels out from its context of production. Occasionally, those choices are brought to the front again, as when (to turn to Espeland and Sauder) U.S. News tweaks its influential law school rankings, but successful quantification projects tend to hide their assumptions - and the extent to which those assumptions remain hidden is perhaps itself a measure of the success of the project. Science studies scholars have long identified infrastructure through its tendency to recede into the background unless and until it breaks down (e.g. Star 1999). To the extent that indicators and measures serve as infrastructure of government and market, they do so best when their own epistemological infrastructures function invisibly.

Somewhat surprisingly, neither Merry nor Barman attend much to the technologies through which quantification is built. Although Merry usefully names the tendency for "data 
inertia" to occur, and Barman talks about valuation "devices", neither book emphasizes the bureaucratic or computational technologies that make possible the measures and indicators under discussion.

And perhaps because they are both focused on somewhat older modes of quantification, neither book addresses the technological advances that promise a continual revolution in our capacities to measure. O'Neil, Neff \& Nafus, and Lupton, by contrast, all focus their gazes on the coming storm. We discuss these books in more detail in the next section, but here we note that studies of the quantified self movement and big data-fueled decisionmaking algorithms in arenas like finance and employment all highlight the immense new quantities of data being produced as parts of other technological and bureaucratic transformations.

"Data inertia" may keep human rights advocates from pinning down their preferred measures of gender violence or human trafficking, but the opposite process - a "data avalanche," say? - is opening up space for a cornucopia of new forms of quantification. Without tackling the question directly, Merry and Barman's work helps us think through how this data avalanche will be channeled into particular measures, and stabilized (or not) around the political projects of particular expert communities and entrepreneurs.

\section{What Does Quantification Do?}

The forces shaping the production and stabilization of new forms of quantification matter because quantification itself matters. That is, there would be little reason to care about how numbers are produced absent evidence that such numbers had the potential to powerfully alter the trajectories of individuals, organizations, and fields. We consider the range of effects identified by authors, starting from the self and proceeding up in scale. 
In Self-Tracking and The Quantified Self, Neff \& Nafus and Lupton directly tackle the question of how quantification of everyday behaviors changes our subjective experiences, as do many of the authors in the edited volume Quantified. Self-Tracking is the ideal entrance into this world for those unfamiliar with the wide array of new tools that have become available for monitoring our steps, our meals, and our very heartbeats. Neff \& Nafus usefully distinguish the explicit "Quantified Self" movement (which has all the features of an emergent community or social movement, from local meet-ups to conferences) from the broader terrain of personal data collection (lower-case "quantified self"). With chapters on the politics of data, how people use data, the self-tracking industry, self-tracking and medicine, and the future, they provide a broad and readable overview of phenomena from step counters to glucose monitors to activity trackers.

Lupton's Quantified Self covers similar territory, but takes a more theoretical approach, framing self-quantification in terms of actor-network theory and Foucauldian governmentality. Quantified, an edited volume, is less synthetic than the other two but more deeply empirical, with a number of interesting case studies of how "biosensing technologies" (a phrase Nafus consciously deploys over “quantified self") are created, governed, and used.

As all three of these books are focused on understanding a particular family of new quantification technologies, they share a focus on an overlapping set of questions. One is how self-tracking affects the self. Neff \& Nafus show that people use self-tracking to achieve a variety of goals - to monitor and evaluate themselves (was I productive today?), to elicit sensations (how do I feel at a particular glucose level?), to satisfy aesthetic curiosity (what patterns can I see in a map of my bike rides?), to debug a problem (what foods trigger my migraines?), and to cultivate habits (can I hit 10,000 steps a day?). 
More deeply, though, a recurring question runs throughout these books: are the numbers liberating or disciplining? The Quantified Self movement assumes the former, and in some cases there seems to be little question that self-quantification improves quality of life. Nafus, who began her project as a self-quantification skeptic, describes her chastened reaction when a colleague pointed out that knowing one's glucose level can mean life or death for a diabetic. Like Nafus, sociologists are likely to start with some doubt—with fear that the technologies that we use to monitor ourselves for fun or out of curiosity produce data that companies use to sell us products, and that employers use to constrain our actions. And of course self-tracking enables Foucauldian self-discipline of new intensity, as we map our use of work time minute-by-minute, or exercise like our health insurance discount depends on it.

The interesting question these books touch on, though cannot fully answer, is how we encourage more of the liberating use of quantification and less of the controlling. In a Quantified chapter on health privacy, philosopher Helen Nissenbaum and cognitive scientist Heather Patterson propose the concept of "contextual integrity": a new set of standards in architecture, law and policy that differentiate between contexts in which one should expect privacy, and others in which the sharing and sale of data can be assumed or conducted with permission. While we agree that shared standards around our right to privacy and use of our number would be very welcome, it is hard to see how we get there from here, as government decisions happen slowly and technological change is rapid. Perhaps a Bill of Data Rights could help us envision such standards.

Of course, it is also possible that self-quantification produces a subject who is neither liberated nor enslaved, but simply different. The solipsism of constant attention to one's numbers creates a self-involved self — one who engages less with the external world because of expanded 
access to the internal one. Anthropologist Jamie Sherman, in another of Quantified's chapters, asks whether self-quantification changes the perception of experience in a way analogous to the one Walter Benjamin described in "The Work of Art in the Age of Mechanical Reproduction." We stop observing experiences in their uniqueness, situated in time and place, and begin seeing them as abstractions represented first and foremost through the data they produce. This may be too much weight to give to the app that calculates how far one has run, but the analogy is interesting.

Self-quantification is generally a freely chosen activity—at least until your life insurance provider requires it. But as individuals we also interact with, and react to, many other numbers that impact our lives. Weapons of Math Destruction are one new and pernicious form these can take. In this widely reviewed and very readable book, mathematician Cathy O'Neil walks us through numerous examples of models for decisionmaking that are opaque, damaging, and scalable - the characteristics she says define Weapons of Math Destruction (WMDs).

The proprietary LSI-R (Level of Service Inventory-Revised) model, for example, predicts a prisoner's chances of recidivism from a questionnaire. It uses secret methods and is grounded in factors strongly associated with race and other forms of disadvantage. When the numbers produced by WMDs lead to decisions that affect our lives, we are forced either to respond to the model (as when we try to raise our credit score) or are trapped without recourse (if the model is fully opaque). WMDs often make decisions based on statistical associations that are nevertheless not causal—poor credit does not itself cause people to perform worse as employees. Or they reflect structural inequalities — prior arrests are associated with neighborhood, which in turn is associated with race; their accuracy as a predictor of recidivism may have nothing to do 
with individual culpability. Pervasive WMDs may require constant low-level attention: are our web-browsing habits telling Amazon it should offer us high prices, or lower ones?

Engines of Anxiety, in which Espeland \& Sauder tackle the U.S. News \& World Report law school rankings as a much simpler yet deeply transformative form of quantification, offers useful concepts for thinking about individual effects as well: numbers provoke reactivity and reshape cognitive maps. Law school deans, professors, alumni, current students, applicants—all consume the same rankings, come to understanding their professional world through their lens, and make decisions accordingly. Some of those consumers (applicants) use the numbers as a guide to action but have little recourse over what they are; others (deans), however, spend much of their energy trying to change them. For the deans and others who live and die by the numbers, "fear of falling" comes to dominate their experience; few subject to this regime think the numbers have improved it.

Still, while the general view is bleak, Espeland \& Sauder allow a little room for agency: individuals can construct narratives around the numbers that tell a different story ("we chose to embrace our mission of providing opportunities to underserved students") than the purely quantitative one ("we fell in the rankings"). And their comparison of law schools with business schools suggests that when competing numbers exist—business schools refer to several sets of rankings, not just one-individuals may choose to focus on the numbers that fit their selfidentity, or that play to their strengths.

The effects of numbers, of course, are not just felt at the individual level. They also affect communities, organizations, and fields. Even self-quantification is experienced by many as a social phenomenon, whether in the original Quantified Self movement (complete with meetups and conferences), in the "SNPNet" forum that collectively puzzles over genotyping results to 
improve treatment protocols for chronic illness (as described by sociologist Mette Kragh-Furbo and colleagues in another of Quantified's chapters), or in the online communities of Uber drivers who puzzle out how to beat the algorithms and maximize their pay.

Espeland \& Sauder are particularly strong on showing the organizational and field-level effects of law school rankings, and on identifying the mechanisms through which such changes take place. Rankings, they argue, have several effects on law schools: "they transform power relations within schools, day-to-day organizational practices, and the ways professional opportunities are distributed" (7). By giving individuals (prospective students, mostly) more information about schools - if a flattened, one-dimensional kind of information - they redistribute power from the schools to the applicants. This altering of power dynamics is one reason law schools may object so strongly to their use. Rankings also give more power to the parts of the organization that produce the inputs into the numbers: the job placement office, or the admissions office. Employees of those offices are also at more risk of consequences, though, should they fail to meet these new expectations.

Within the organization, practices emerge and relationships change in response to rankings. Marketing becomes more important (both to generate applications and to improve reputational scores), deans spend a substantial amount of energy on managing rankings, and colleagues at other schools become competitors. And across the field, the distribution of status and opportunities changes. Reducing diverse schools to a single measure means homogenizing them, and allowing fewer niches for specific types of excellence or the pursuit of goals that, while in conflict with the rankings, might themselves be worthwhile. Small differences are also amplified; applicants take seriously the idea that their life trajectory might be meaningfully worse if they attend \#24 instead of \#21, and so the schools must as well. 
Finally, beyond organizations and fields, numbers also have effects on other numbers, and even on themselves. Numbers currently established affect what we will measure in the future, as Merry's concept of "data inertia" suggests. For example, since the decision was made to exclude the value of domestic labor from Gross National Product some eighty years ago, housework has never really been accounted for, despite its clear economic contribution. And numbers affect their own future meaning, as they produce "reactivity" (as Espeland \& Sauder describe it) among those subject to them and come to measure something different. O'Neil's "pernicious feedback loops" are the most extreme version of this: a model predicts a prisoner from a poor, highly policed neighborhood will be more likely to be rearrested and keeps him in prison longer. When he is out and returns to his neighborhood, he is more likely to reoffend, having been in the prison environment longer. When he does, the model's prediction—offenders from this neighborhood will reoffend—is proven correct, and the model reinforced.

Yet as significant as these effects can be, a great deal of work has to be done in order to make them happen in the first place. Barman shows us how hard value entrepreneurs work to gather support for their numbers and convince others to use them. Merry demonstrates how human trafficking indicators largely sit on the shelf because no audience for them has been created. Espeland \& Sauder choose their number because it is such an effective one, but what makes it effective? Their business school comparison suggests the dominance of a single ranking over law schools is one factor. We might hypothesize other reasons law schools seem particularly prone to fetishizing rankings. In $\mathrm{PhD}$ programs, for example, specific faculty members play a significant part in applicant decisions; it is clearer that the school ranked three places higher is not, in fact, the better choice if that school has no faculty you want to work with. Plenty of questions remain about not just whether numbers matter, or how they matter, but when. 


\section{How Should We Govern Numbers?}

This leads us, then, to the ethical questions. Numbers play a large and increasing role in governing social life. But how should we, in turn, govern the numbers? Must we simply accept their proliferation in whatever form as inevitable, or are there better and worse ways they can be used? At some level, all the works we discuss are interested in this question, though some address it directly and others allow us to draw our own conclusions.

O'Neil's own algorithm for identifying Weapons of Math Destruction is among the most explicit. She provides a rubric for deciding whether any given model for decisionmaking deserves our condemnation. O'Neil tells us to ask three questions: Is it opaque? Does it scale? And can it do damage? We might quibble with "moneyball" approach to baseball for its lack of romance or inability to measure a player's potential. But the numbers that go into such models (runs batted in, strikeouts) are publicly available, there are clear causal relationships between those numbers and the desired outcome (wins), and a steady stream of new data allows us to test and improve the models. O'Neil gives them the thumbs up.

The LSI-R recidivism model, by contrast, is opaque. What goes into it? How are scores generated? It scales: twenty-four states use it at present, according to O'Neil. And it has the potential to do serious damage, keeping someone locked up for longer based on factors they have no control over. O'Neil recommends some WMDs simply be abandoned (like value-added models of teaching, which may be useful in understanding aggregate performance but are terrible for evaluating individual teachers), and others be subjected to algorithmic audits, which would prevent models from making predictions based on illegal or unethical factors, or their proxies. These recommendations are appealing, although we suspect defining "opaque" or "damage" is 
harder than it might initially seem. Moneyball models are still proprietary, after all, even if the inputs are clear, and a bad model can clearly damage a player's career.

Moreover, transparency, which O’Neil presents as an unqualified good, can actually be a double-edged sword. U.S. News rankings are totally transparent, but it is that very transparency that encourages reactivity, as Espeland \& Sauder show. Sometimes this is just what is intended. Many numbers are meant to incentivize certain behavior, and if their calculation is mysterious, they can't produce the desired effects. As long as numbers have consequences, though, people will try to game them, and transparency facilitates this.

Robert Saldin's short but excellent case study, When Bad Policy Makes Good Politics: Running the Number on Health Care Reform, provides one such example. The CLASS (Community Living Assistance Service and Supports) Act, designed to improve long-term care insurance, was included in Obamacare purely to improve its Congressional Budget Office (CBO) score. Users would pay premiums from day one, but not be eligible for benefits for five years. This meant that over the 10 -year period that went into the CBO score, CLASS would look extremely lucrative, offsetting some of the Affordable Care Act's other costs. Yet over a 40-year period, it would cost $\$ 2$ trillion. The program was included in order to make the numbers work despite the fact that it was completely unsustainable. Despite becoming law, CLASS was never implemented, because the Obama administration couldn't find a way to make it remotely feasible.

The transparency of the CBO process is precisely what made this gaming possible. Opacity, despite its other drawbacks, may be the only way to quantify without changing behavior. But opacity is no solution either when the actual purpose of quantification is to change behavior - in this case to encourage more financially responsible legislation. The trick is to 
create quantification techniques that encourage the desired behavior changes (attention to longterm costs) but minimize the opportunities for gaming (with legislation that produces the desired numbers but undermines their intent). This is a needle that is truly difficult to thread.

A few normative lessons do emerge from these books. Merry, for example, argues convincingly that counts and ratios, despite their relative simplicity, generally stay closer to the intent of their creators than indicators do, because counts and ratios "require less interpretive work than composites and are less vulnerable to the twists produced by long interpretive chains" (217). Rankings, Espeland \& Sauder remind us, are a zero-sum game: “one person’s climb requires someone else's descent" (130). And the self-quantification books highlight the positive effects of numbers, as well as their constraints.

More generally, we would also emphasize that we turn to numbers to make decisions that are already being made through other means. Even forms of quantification that seem ethically questionable may be better than the alternative that actually exists in the world. New Jersey recently "replaced bail hearings with algorithmically informed risk assessments" (Livni 2017). While O'Neil's critiques could readily be applied to the "Public Safety Assessment" formula, in its first six months of use New Jersey's jail population fell by roughly 20\% (Kelly 2017). Given how broken the bail system is and how much it already disadvantages poor defendants, even a biased algorithm seems like a step in the right direction. Subjective or expertise-based decisionmaking is no panacea, and while quantification often crystallizes existing problems, fixating on it can be a distraction, rather than a solution. These examples all highlight the need to focus on the how of quantification as much as the mere fact of it - as with any other powerful tool, the effects depend on intentions and implementation. 


\section{How Should Quantification Be Studied?}

Studying quantification is a growth industry. Alongside the rise of data science in industry, academia, and government, we see a parallel emergence of new dedicated venues for examining these transformations, including the Data \& Society Research Institute and journals like Big Data \& Society. What can we learn from this recent generation of quantification studies to inform the path ahead?

We see three rough approaches represented here: charting a single (often new) quantified phenomenon, comparing quantification projects (often bounded to a single field or domain), and tracing a particular historical case where quantification took center stage. Each of these approaches offers particular advantages and characteristic weaknesses.

Tracing a single case, as in Saldin's history of the $\mathrm{CBO}$ and health reform, or Espeland and Sauder's study of the effects of law school rankings on legal education, may be most useful for thinking about the mechanisms through which relatively influential forms of quantification exercise that influence. The nitty gritty of the policymaking process, and the ability to compare organizational practices before and after the emergence of law school rankings, provide rich within-case details that illuminate how particular numbers do their work. These cases are less useful, however, when it comes to specifying scope conditions. This problem is not unique to single case studies of quantification, but it does impel us to reach for more.

Explicitly comparative projects, like Barman's work on social value, Merry's research on human rights indicators, and O'Neil's analysis of destructive algorithms, offer important insights about the conditions under which quantification matters. While the USNWR rankings clearly altered legal education, Merry's work shows how little oomph some rankings possess. Absent a comparative lens, we collectively tend to fixate on the most prominent forms of quantification 
(like GDP or presidential approval polls) and miss the forest of much less potent indicators that surround them. Comparing across cases within a domain reminds us of the mundane organizational and political factors that shape the reception of quantification by highlighting the presence and absence of powerful, motivated actors pushing or resisting forms of quantification. Comparing across domains, as O'Neill does, productively highlights the distinctive aspects of contexts that may inhibit or privilege quantification, showcasing why (for example) algorithmic decisionmaking is relatively uncontroversial in credit, but fiercely fought in education.

Finally, studies of new quantified phenomena help to sharpen our conceptual categories for thinking about what quantification really is. Quantification is not a single, unified process. Work that explores the boundaries of numbers, data, and measurement helps to flesh out our vocabulary - from indicators and rankings, to models and algorithms, and now big data and biosensing, and so on. Indeed, one of the clearest takeaways from these books, read as a group, is the blurriness of "quantification", and the need for conceptual categories that will help us unpack it. What qualities are specific to rankings, or indicators, or models, or algorithms? What does quantification share with related concepts like commensuration or categorization?

Some phenomena that, when one is primed to think of them in terms of quantification, seem to fit right in, aren't quite examples of quantification at all on closer inspection. Nafus discusses her decision to talk about "biosensors" rather than the "quantified self"; when one is interested in understanding, for example, how heart rate monitors change human experience, which of these terms directs our attention to the most relevant aspects of the new technology? Or when, as in Barman's work, valuation of an organization's social impact produces not a number but a binary yes/no, are we still talking about quantification? Maybe not, and yet the process of producing that binary seems quite similar to that of calculating of social return on investment. 
Following Barman, then, we see promise in distinguishing more explicitly among the intended uses of numbers - their "communicative goals" (or, we would add, "performative goals"). Law school rankings have both an explicit purpose and an intended audience; those shape the process of quantification and its effects. So do human rights indicators, and CBO scores, and activity trackers. Though numbers in the wild may certainly be used in ways their creators never anticipated, clearer and more explicit attention to the purposes of both producers and consumers would help us think more clearly about variation in the quantification process across contexts.

There is also plenty of room for more comparative work that explores how numbers are produced (the same number in different organizational or social contexts, or different numbers in similar contexts) and on when and how they have effects (including comparisons of failure and success, as well as variation across successful cases). And there is certainly more space for synthetic work and theory to give us a common way of talking about these diverse phenomena, and to build insights across a broad range of quantification processes. The qualitative study of quantification may, at the moment, be producing a significant body of new work. But for producing a coherent sociology of quantification, it is still early days. 


\section{Bibliography}

Espeland, Wendy Nelson, and Mitchell L. Stevens. 2008. "A Sociology of Quantification." European Journal of Sociology 48:401-36.

Hacking, Ian. 1982. "Biopower and the Avalanche of Printed Numbers." Contemporary Sociology 5:279-95.

Hecht, Gabrielle. 1998. The Radiance of France: Nuclear Power and National Identity after World War II. Cambridge: MIT Press.

Kelly, Albert B. 2017. "Six Months In, N.J. Bail Reform Is Working." Available at http://www.nj.com/opinion/index.ssf/2017/07/six_months_in_nj_bail_reform_is_working _opinion.html (last accessed 4 September 2017).

Livni, Ephrat. 2017. "In the US, Some Criminal Court Judges Now Use Algorithms to Guide Decisions on Bail." Available at https://qz.com/920196/criminal-court-judges-in-newjersey-now-use-algorithms-to-guide-decisions-on-bail/ (last accessed 4 September 2017).

Porter, Theodore. 1995. Trust in Numbers. Princeton: Princeton University Press.

Star, Susan Leigh. 1999. "The Ethnography of Infrastructure." American Behavioral Scientist 43:377-91. 